\title{
InP-nanocrystal monolayer deposition onto Silicon-on-Insulator structures.
}

\author{
Gino Priem*, Iwan Moreels ${ }^{\ddagger}$, Pieter Dumon*, Zeger Hens ${ }^{\ddagger}$, Wim Bogaerts*, \\ Dries Van Thourhout*, Geert Morthier*, and Roel Baets* \\ * Photonics Research Group, Dept. of Information Technology, Ghent University - IMEC, Belgium \\ $\ddagger$ Physical Chemistry Laboratory, Dept. of Inorganic and Physical Chemistry, Ghent University, Belgium \\ Gino.Priem@UGent.be - Iwan.Moreels@UGent.be
}

\begin{abstract}
We demonstrate, for the first time, the deposition of an InP nanocrystal monolayer onto a processed Silicon-onInsulator substrate containing nanophotonic devices. From the first results, propagation losses of $1.7 \mathrm{~dB} / \mathrm{mm}$ were obtained for wires coated with InP nanocrystals compared to $0.26 \mathrm{~dB} / \mathrm{mm}$ for the uncoated case.
\end{abstract}

\section{INTRODUCTION}

In recent years, the interest in Silicon-on-Insulator (SOI) as a platform for photonic integrated circuits has grown considerably. In this high-contrast systems, basic components such as low-loss photonic wires and bends, power splitters, MMI's and Mach-Zehnder interferometers have already been demonstrated [1], [2], but also more advanced structures such as different types of ring resonators, grating couplers, spot-size converters... [3].

However, for some dedicated applications such as nonlinear functionalities and optical amplification, the properties of standard semiconductor systems like the SOI platform may prove to be insufficient. A heavily investigated road to solve these shortcomings is that of heterogeneous integration, in which different material systems are combined to provide all the required functions. An example of this is active-passive integration of Silicon and III-V by BCB bonding [4].

Another possibility is providing additional functionality directly onto the core material system by additional depositions. As the propagating modes of submicron structures such as photonic wires have a considerable part of their field profile outside the structure, they can interact with the material system deposited onto the structure. Inside resonant structures such as ring resonators, this interaction can even be further enhanced. Excellent candidates for such depositions are semiconductor nanocrystals, as considerable tailoring of electrical and optical properties is possible by changing the particle size [5]. Monolayer deposition of nanocrystals is however very difficult for submicron structures such as photonic wires, because of their comparable dimensions in height and width.

In this paper, we describe the deposition of InP nanocrystals onto Silicon-on-Insulator nanostructures. The presence of the nanocrystal coating was verified by scanning electron microscopy (SEM) and atomic force microscopy (AFM). From the first results, propagation losses were derived for the coated photonic wires.
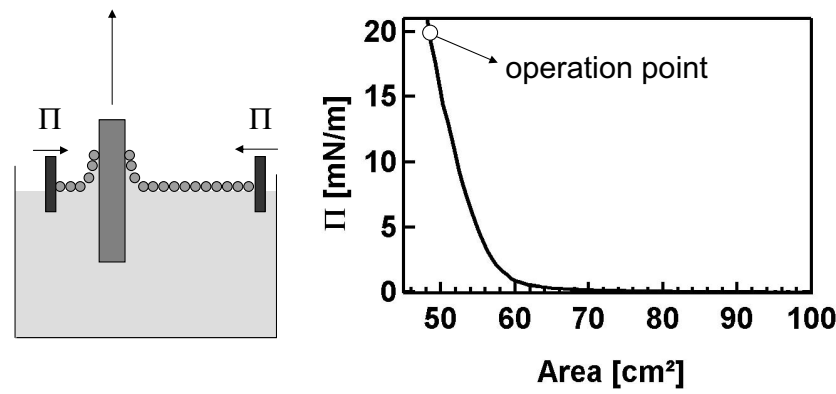

Fig. 1. Deposition of a nanocrystal monolayer onto an SOI substrate: after the SOI sample is placed into the water, InP|PMS nanocrystals are added. Before deposition on the substrate, the nanocrystals are compressed until they form a close-packed film (surface pressure $\Pi$ of $20 \mathrm{mN} / \mathrm{m}$ ). At this point, the sample is slowly drawn out of the water with a nanocrystal monolayer on top.

\section{FABRICATION}

Different types of submicron structures were created on $200 \mathrm{~mm}$ SOI wafers with a thickness of the Silicon layer of $220 \mathrm{~nm}$ and a burried oxide of $1 \mu \mathrm{m}$. A deep UV lithography stepper with a $248 \mathrm{~nm}$ illumination wavelength defines the patterns in the resist. A dry etching process is then used to transfer the patterns of the resist into the Silicon layer. A full overview of the processing steps can be found in [3]. These processes are in fact CMOS processes, which have been adapted for the fabrication of photonic circuits.

Colloidal InP nanocrystals (Q-InP) with an organic capping of tri-octyl phosphine oxide (TOPO) were synthesized according to established literature methods [6]. After synthesis, monodisperse colloids $(\sigma<10 \%)$ were obtained by sizeselective precipitation. In this way, nanocrystals with sizes $2-$ $5 \mathrm{~nm}$ can be fabricated. Prior to deposition, the TOPO ligand was exchanged for pentamethylene sulfide (PMS) offering a short and rigid capping. To deposit these nanocrystals onto the SOI wafer, the Langmuir-Blodgett method [7] was used (Figure 1): a SOI sample is placed into water container, after which the nanocrystals are added. The Q-InP|PMS particles spread on the water surface and can be forced into a close packed film by compressing the surface area. An example of the required surface pressure $\Pi$ as a function of nanocrystal film area is also shown in Figure 1. At the working pressure 
of $20 \mathrm{mN} / \mathrm{m}$, the Q-InP|PMS film is transferred to the SOI sample by pulling the sample out of the water at a rate of $1 \mathrm{~mm} / \mathrm{min}$.

\section{VERIFICATION}

To verify the deposition of a InP nanocrystal monolayer on the samples, SEM imaging was used. An example of a photonic wire before and after deposition of the nanocrystals is shown in Figures 2 and 3. As can be seen, the obtained nanocrystalline layer is still not completely uniform, however a good coverage of the complete sample was already obtained.

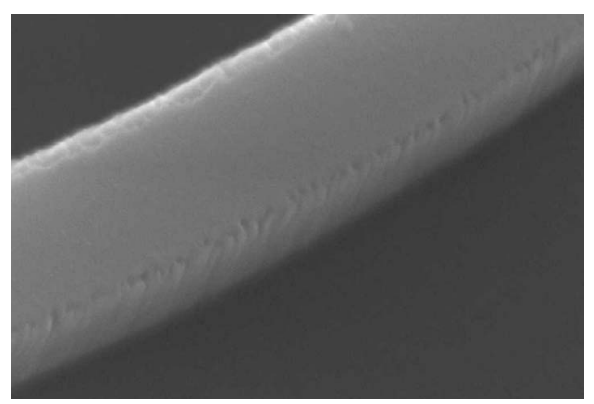

Fig. 2. Uncoated photonic wire. Note the side wall roughness due to the dry-etch process.

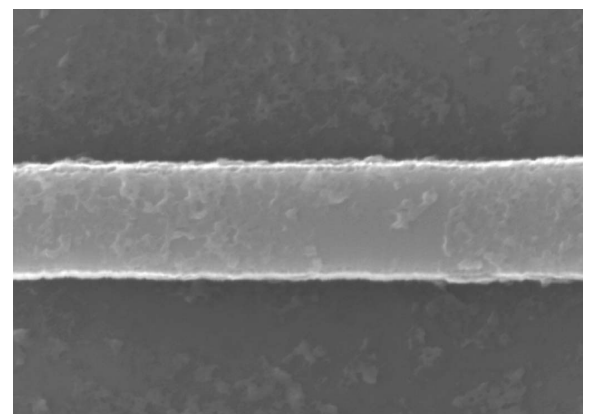

Fig. 3. Coated photonic wire. A relatively good coverage of the wire has been obtained.

As these are only first results, improvements in the applied deposition method will surely allow to overcome these nonuniformities. AFM measurements have also been performed, confirming the deposition of object with sizes of several $\mathrm{nm}$, i.e. the nanocrystals.

\section{WIRE LOSSES}

After the verification of the nanocrystal deposition, wire losses were calculated based on the comparison of transmission measurements for wire lengths of $250,500,1000$ and $2000 \mu \mathrm{m}$. The nominal wire width before coating was approximately $540 \mathrm{~nm}$. The results are shown in figure 4 .

On average, a wire loss of $1.71 \mathrm{~dB} / \mathrm{mm}$ was obtained with an standard deviation of $0.3 \mathrm{~dB} / \mathrm{mm}$. Compared to the $0.26 \mathrm{~dB} / \mathrm{mm}$ loss in the uncoated case, this value is acceptable for first experiments.

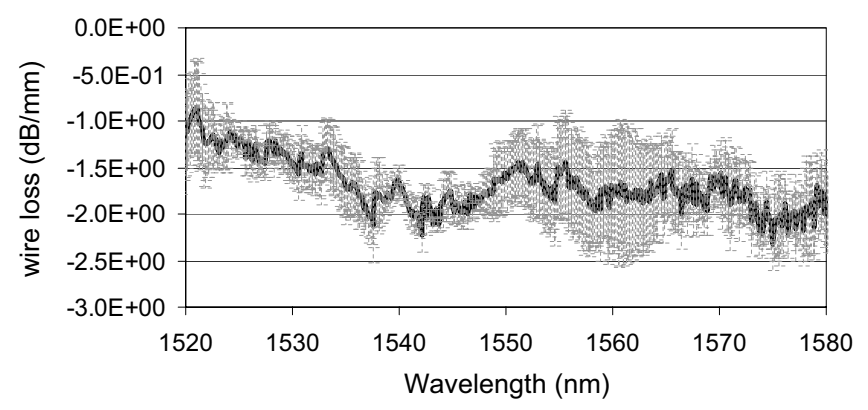

Fig. 4. Calculated wire losses of a coated sample for different wavelengths. The standard deviation is also shown.

\section{CONCLUSION}

The deposition of InP nanocrystals onto Silicon-on-Insulator nanophotonic structures have been reported. The coating of standard semiconductor structures with nanocrystals may provide additional functionalities such as nonlinear effects or optical amplification potential directly onto the wafer. In this way, costy heterogeneous integration could be avoided. Deposition of a nanocrystal monolayer onto nanophotonic components is however difficult due to the comparable dimensions in width and height of these structures. To solve this problem, the Langmuir-Blodgett approach was used.

Verification of the presence of these nanocrystals onto the nanostructures was done by means of SEM and AFM measurements. First results show a relatively good coverage of the complete sample. However the uniformity of the deposited monolayer was not yet optimal. Despite this non-uniformity, wire losses of only $1.71 \mathrm{~dB} / \mathrm{mm}$ were obtained, indicating that the roughness of the deposited layer is still acceptable.

\section{ACKNOWLEDGMENT}

This work was supported by the EU through the NoE ePIXnet project. Part of this work has been performed in the context of the Belgian IAP PHOTON Network (IAP V/18).

G.P. and W.B. thank the Flemish Fund for Scientific Research (FWO-Vlaanderen) for financial support. I.M. and P.D. thank the Institute for the Promotion of Innovation through Science and Technology in Flanders (IWT-Vlaanderen) for a scholarship.

\section{REFERENCES}

[1] P. Dumon et al., "Basic photonic wire components in silicon-oninsulator,' in Group IV 2005, Belgium, 92005 (accepted).

[2] — , "Low-loss photonic wires and ring resonators fabricated with deep uv lithography," IEEE Photon. Technol. Lett., vol. 16, no. 5, pp. 13281330, May 2004.

[3] W. Bogaerts et al., "Nanophotonic waveguides in silicon-on-insulator fabricated with cmos technology," J. Lightwave Technol., vol. 23, no. 1, pp. 401-412, January 2005.

[4] G. Roelkens et al., "Ultra-thin bcb bonding for heterogeneous integration of iii-v devices and soi photonic components," in ECIO 2005, France, 4 2005 (accepted).

[5] V. Degiorgio et al., "Optical nonlinear properties of semiconductor nanocrystals," Adv. in Physics, vol. 47, no. 3, pp. 447-210, 1998.

[6] A. Guzelian et al., "Synthesis of size-selected, surface-passivated inp nanocrystals," J. Phys. Chem., vol. 100, no. 17, pp. 7212-7219, 1996.

[7] M. Petty, Langmuir-Blodgett films, an introduction, Cambridge University Press, 1996. 